Case Report

\title{
Rapid Evaluation of Mutant Exon-11 in c-kit in a Recurrent MCT Case Using CD117 Immunocytofluorescence, FACS-Cell Sorting, and PCR
}

\author{
Dettachai Ketpun, ${ }^{1,2}$ Achariya Sailasuta, ${ }^{1}$ Prapruddee Piyaviriyakul, ${ }^{2}$ \\ Nattawat Onlamoon, ${ }^{3}$ and Kovit Pattanapanyasat ${ }^{3}$ \\ ${ }^{1}$ STAR, Molecular Biology Research on Animal Oncology, Department of Pathology, Faculty of Veterinary Science, \\ Chulalongkorn University, Bangkok 10330, Thailand \\ ${ }^{2}$ Biochemistry Unit, Department of Physiology, Faculty of Veterinary Science, Chulalongkorn University, Bangkok 10330, Thailand \\ ${ }^{3}$ Office for Research and Development, Faculty of Medicine Siriraj Hospital, Mahidol University, Bangkok 10770, Thailand
}

Correspondence should be addressed to Achariya Sailasuta; achariya.sa@chula.ac.th

Received 4 June 2013; Accepted 5 August 2013

Academic Editors: J. Lakritz, F. Mutinelli, and S. Stuen

Copyright (C) 2013 Dettachai Ketpun et al. This is an open access article distributed under the Creative Commons Attribution License, which permits unrestricted use, distribution, and reproduction in any medium, provided the original work is properly cited.

A 13-year-old, poodle-mixed, male dog was referred to the oncology unit in our faculty's small animal teaching hospital with the problem of rapid recurrent MCT. The owner and the veterinarian would like to use a tyrosine kinase inhibitor (TKI) for the dog. Therefore, fine-needle aspiration (FNA) was performed to collect the MCT cells and these cells were submitted to our laboratory for the detection of internal-tandem-duplicated (ITD) mutation of exon-11 in $c$-kit, prior to the treatment. The aim of this paper is to demonstrate the use of combinatorial protocol for the rapid evaluation of ITD mutation in MCT cells harvested by FNA. However, there was no ITD-mutant exon-11 that had been observed in this case.

\section{Introduction}

Canine cutaneous mast cell tumors (MCT) are the second most skin tumors found in dogs. The incidence is probably medium to high in some breeds, such as boxer, bull dog, pug, poodle, labrador retriever, and golden retriever. In general, all MCT patients required an aggressive diagnosis and therapy because the progression of disease is very rapid [1]. Among obscured tumorigenesis of MCT, however, there has been much information from various studies showing that the mutation of exon-11, called internal-tandemduplication (ITD), of proto-oncogene, $c$ - $k i t$, is involved in MCT formation. Principally, $c$-kit is a protein-coding gene responsible for KIT (CD117) formation and it is usually expressed in many cell species, such as normal mast cells, melanocytes, and Purkinje cells including MCT cells [2].

KIT is a member of the receptor-tyrosine-kinases class III (RTKs class III). It consists of three functioning domains, extracellular (ectodomain), transmembrane (TM), and intracellular domains, respectively [3]. The intracellular domain is further separated into two subsidiary portions; juxtamembrane encoded by exon-11 and kinase domains encoded by the remaining exons. The function of KIT is triggered when the ectodomain binds to the specific ligand, stem cell factor (SCF), followed by KIT dimerization and crossautophosphorylation to tyrosine residues on the dimerized KITs. The consequence is to activate the downstreamsignaling cascades, which are responsible for the growth and proliferation as well as antiapoptosis of mast cells. In case of MCT, ITD mutation of exon-11 results in the abnormally autonomous KIT dimerization without any specific ligand binding. This mechanism leads to uncontrollable autophosphorylation followed by MCT formation. Nevertheless, this type of mutation also affects MCT therapy when a tyrosine kinase inhibitor (TKI), such as SU-11654, is used [4]. Therefore, the detection of ITD-mutant exon-11 is clinically 
important for MCT diagnosis and therapeutic planning. However, a utilization of MCT cells collected by FNA for the detection of ITD-mutant exon-11 has not recently been reported anywhere.

The objective of this paper is to report the employment of CD117 immunocytofluorescence and FACS-cell sorting for rapid identification and isolation of MCT cells harvested by FNA. In addition, we also demonstrate the usage of these cells to diagnose ITD-mutant exon-11 by PCR in a recurrent MCT case.

\section{Case Presentation}

A 13-year-old, poodle-mixed, male dog was referred to the Oncology Clinic, Small Animal Teaching Hospital, Faculty of Veterinary Science, Chulalongkorn University, with the recurrent MCT-grade II (based on Patnaik histopathologic grading) at the neck and the dorsomedial of the left palmar (Figure 1). The dog was undertaken a surgical removal with prophylactic chemotherapy along the year. However, the treatment was ineffective and the progression of the disease was rapidly advanced. Finally, the owner and the responsible veterinarian planned to use a tyrosine kinase inhibitor (TKI) in this case. However, the owner was unwilling to repeat a skin biopsy for his dog. Hence, the previous tissue section was sent to our laboratory for histopathology reevaluation grounded on the novel 2-tier histopathologic grading system [5] and the veterinarian exploited FNA to aseptically collect the cell sample from the mass at the left palmar (Figure 1) for investigating ITD-mutant exon-11.

To identify MCT-cell population, a few drops of neoplastic cells from FNA were smeared on a clean silane-coated glass slide. The cells were then preserved in $4^{\circ} \mathrm{C}$ cold acetone for a minute and they were incubated by $1 \%$ bovine serum albumin (BSA) for 30 minutes at room temperature to block nonspecific proteins. The tumor cells were further incubated with PE-conjugated mouse monoclonal anti-human CD117 antibodies (Clone Y.B5.B8, Becton and Dickinson, USA) for 30 minutes at the concentration of $1: 100$ at room temperature in a dark chamber. The specificity of the antibodies was already approved by our laboratory in our previous study. The MCT cells were counterstained by $4^{\prime}, 6$-Diamidino2-Phenylindole (DAPI, Invitrogen, USA), and they were visualized under a fluorescent microscope with $575 \mathrm{~nm}$ light source. In this study, we also used normal mast cells and histiocytoma cells prepared by the same method, as the positive and negative controls, respectively.

For ITD-mutation assessment, the neoplastic cells were firstly purified using FACS-cell sorting. Briefly, the cells previously harvested by FNA were resuspended in $500 \mu \mathrm{L}$ sterile PBS. The erythrocytes were eradicated from the cell suspension by adding $10 \mathrm{~mL}$ of BD Facs lysing solution for 10 minutes (Becton and Dickinson, USA). Further, the cell suspension was centrifuged at $4,000 \times \mathrm{g}$ for 5 minutes and the supernatant was discarded after centrifugation. The cell pellet was then added by $500 \mu \mathrm{L}$ BD FacsPerm solution (Becton and Dickinson, USA) for 10 minutes to increase membrane permeability, and it was washed by PBS twice. The neoplastic

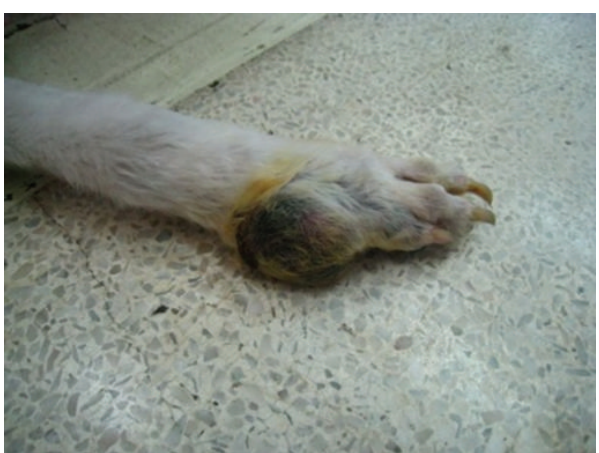

FIgURE 1: The location of MCT mass at the dorsomedial plane of the left palmar of the case in which the MCT cells were harvested from the site.

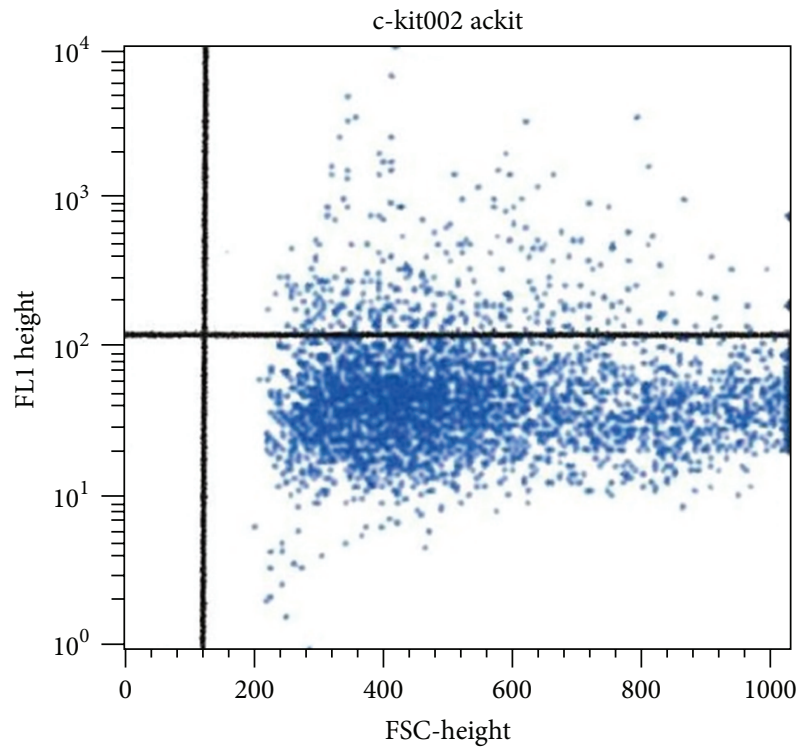

FIgure 2: The dot plot of CD117-immunopositive MCT cells in this case was drawn back by the software. The MCT cells were gated electronically and were harvested from the cells presenting above the baseline, at the quadrant II (upper-right) of dot plot.

cells were incubated, in the dark chamber, by $100 \mu \mathrm{L}$ of PE directly conjugated mouse monoclonal anti-human CD117 antibodies (Clone Y.B5.B8, Becton and Dickinson, USA), at concentration of $1: 200$ for 20 minutes. The cells were washed and centrifuged again at $4,000 \times \mathrm{g}$ for 5 minutes and the supernatant was then decanted. The cell sediment was resuspended in $10 \mathrm{~mL}$ of sterile PBS. Data acquisition was performed using BD-FACScalibur Cell Sorter with BD CellSorting software system (Becton and Dickinson, USA) and the CD117-immunopositive cells were gated from the cells presenting above the threshold line, at the quadrant II of dot plot (Figure 2). The cells were sorted out and harvested into a collecting tube for PCR analysis. The monoclonal IgG was used as the isocontrol for establishing the threshold line on the dot plot. 
The genomic DNA (gDNA) of FACS-sorted neoplastic cells was extracted using the commercial DNA-isolation kit (Mobio, USA) and its concentration was computed using UVspectrophotometry. The forward and reverse primers were designed from $5^{\prime}$ end of exon- 11 and $3^{\prime}$ end of intron-11 of $c$ $k i t$, respectively $[6,7]$. The sequence of forward primer was $5^{\prime}$ CCA TGT ATG AAG TAC AGT GGA AG- $3^{\prime}$ and the reverse primer was $5^{\prime}$-GTT CCC TAA AGT CAT TGT TAC ACG-3', respectively.

The PCR cocktail was prepared in a flat-capped PCR tube (Axygen, USA). $25 \mu \mathrm{L}$ of PCR mixer consisted of $1.5 \mu \mathrm{L}$ of $10 \mathrm{X} \mathrm{KCl} \mathrm{buffer} \mathrm{solution} \mathrm{(DreamTaq,} \mathrm{Fermantas,} \mathrm{USA),} 1.5 \mu \mathrm{L}$ of 10X $\left(\mathrm{NH}_{4}\right)_{2} \mathrm{SO}_{4}$ buffer solution (DreamTaq, Fermantas, USA), $3 \mu \mathrm{L}$ of $20 \mathrm{mM} \mathrm{MgCl}_{2}$ solution (Fermantas, USA), $1 \mu \mathrm{L}$ of $2 \mathrm{mM}$ dNTP solution (Fermantas, USA), $0.5 \mu \mathrm{L}$ of Taq polymerases (DreamTaq, Fermentas, USA), $2 \mu \mathrm{L}$ of $10 \mathrm{mM}$ forward primers solution, $2 \mu \mathrm{L}$ of $10 \mathrm{mM}$ reverse primers solution, $4 \mu \mathrm{L}$ of purified DNA template, and $9.5 \mu \mathrm{L}$ of nuclease-free water (Mobio, USA). The DNA template was amplified in the thermocycler (G-Storm, USA) with the batch of programmatic temperatures of $95^{\circ} \mathrm{C}$ for 5 minutes for initial DNA denaturation; 40 cycles of $95^{\circ} \mathrm{C} 1$ minute for cyclic DNA denaturation, $57^{\circ} \mathrm{C}$ for 1 minute for cyclic DNA annealing, and $72^{\circ} \mathrm{C}$ for 1 minute for cyclic DNA extension; and $72^{\circ} \mathrm{C}$ for 5 minutes for complete DNA elongation [8]. DEPC water was used as the negative control in this study. Meanwhile, gDNA of non-ITD and ITD-mutant exon-11 cells prepared by College of Veterinary Medicine, Michigan State University, USA, was utilized as the normal and positive controls, respectively. In addition, we also used one positive specimen prepared by the same protocols for collating the result with this case.

Ultimately, the amplicons were separated using $2 \% \mathrm{EtBr}$ mixed agarose gel electrophoresis at $100 \mathrm{~V}$ for 40 minutes. The amplicons were visualized by the gel documentation apparatus (Bio-Rad, USA). The information was analyzed by the computerized software system, Quantity One version 4.6.9 (Bio-Rad, USA), and transformed into JPEG-imaging system using Microsoft Paint (Microsoft, USA).

Upon the study results, the histopathologic section was reclassified as the high-grade MCT. Moreover, CD117 immunocytofluorescence clearly ratified the MCT-cell population in the aspirate. All tumor cells strongly exhibited CD117 immunopositivity on their plasma membranes and cytoplasm; meanwhile, their nuclei were stained blue by DAPI, as shown in Figure 3. It is noteworthy to notice that there were some CD117-immunonegative cells presenting in the smear. These cells were not MCT cells as compared to the negative control.

In addition, approximately 400,000 FNA-MCT cells were harvested by FACS-cell sorting. The genomic DNA of these cells was extracted using the aforementioned protocol and its concentration was $156.65 \mu \mathrm{g} / \mathrm{mL}$. There was no ITD mutant exon-11 of c-kit observed in this case (Figure 4). The single band of PCR product in this case was $191 \mathrm{bp}$ compared to the positive control and the positive specimen ( 2 product bands at $191 \mathrm{bp}$ and $250 \mathrm{bp}$, resp.) and the normal control (191-bp).

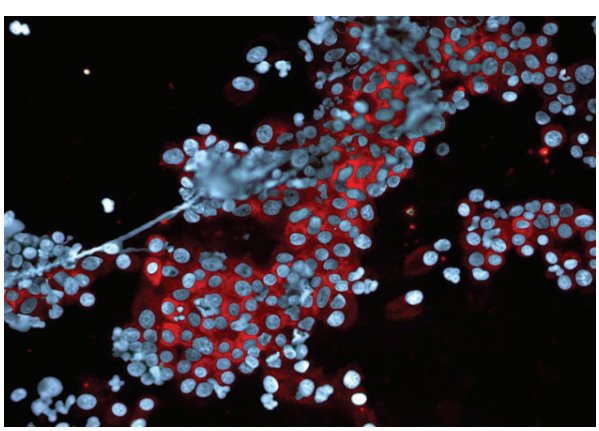

FIGURE 3: CD117 immunocytofluorescence has confirmed MCT subpopulation in the aspirate. The nuclei were stained blue by DAPI. Notably, some cells were non-MCT cells because of lack of CD117 immunopositivity in their cytoplasm and on plasma membranes.

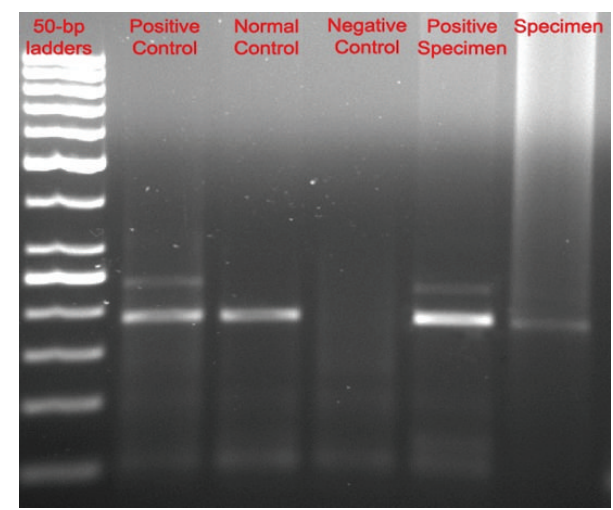

FIGURE 4: The $191 \mathrm{bp}$ of PCR product in this case (in specimen lane) substantially suggested that there was no ITD-mutant exon-1, as well as in the normal control. Notably, the PCR products of the positive control and the positive specimen from the other case consisted of 2 distinct bands at $191 \mathrm{bp}$ and $250 \mathrm{bp}$ (ITD).

\section{Discussion}

The ITD-mutant exon-11 in $c$-kit is seemingly essential in MCT tumorigenesis as this mutation has been widely studied $[3,6,8]$. Moreover, the ITD-mutation of exon-11 also influences a TKI administration. Basically, MCT patients who possess ITD-exon-11, usually respond to a TKI therapy greater than nonmutational patients [4].

In this study, we have demonstrated an alternative protocol for rapid MCT diagnosis. CD117-immunocytofluorescence could enhance our ability for precise identification of MCT cells. Particularly, in high-grade MCT in which the neoplastic cells are usually pleomorphic and their morphology might mimic other neoplastic cells, such as round-cell tumor cells. Moreover, FACS-cell sorting could rapidly facilitate us to purify the MCT population. These MCT cells were a good source of cells which could be utilized to detect the ITD-mutant exon-11, at least in this case. A rapid diagnosis of ITD-mutant exon-11 may help a veterinarian to decide himself on a TKI usage. 
In this case, we supposed that this case should have mutant exon-11 due to an aggressive behavior of the neoplasm. However, ITD-mutant exon-11 was not observed in this case. This might suggest that the mutant exon-11 might not associate with histopathologic grade, but it should associate with the recurrence rate. However, we strongly recommend that a further study based on our combinatorial protocol must be performed in a large population to ensure the advantage of this protocol, before this combinatorial method will be used as a standard protocol for rapid MCT diagnosis in the future.

\section{Conflict of Interests}

The authors would like to clarify that there is no conflict of interests with any financial organization regarding the materials discussed in this paper.

\section{References}

[1] M. M. Welle, C. R. Bley, J. Howard, and S. Rüfenacht, "Canine mast cell tumours: a review of the pathogenesis, clinical features, pathology and treatment," Veterinary Dermatology, vol. 19, no. 6, pp. 321-339, 2008.

[2] M. Morini, G. Bettini, R. Preziosi, and L. Mandrioli, "C-kit gene product (CD117) immunoreactivity in canine and feline paraffin sections," Journal of Histochemistry and Cytochemistry, vol. 52, no. 5, pp. 705-708, 2004.

[3] S. Letard, Y. Yang, K. Hanssens et al., "Gain-of-function mutations in the extracellular domain of KIT are common in canine mast cell tumors," Molecular Cancer Research, vol. 6, no. 7, pp. 1137-1145, 2008.

[4] C. A. London, P. B. Malpas, S. L. Wood-Follis et al., "Multicenter, placebo-controlled, double-blind, randomized study of oral toceranib phosphate (SU11654), a receptor tyrosine kinase inhibitor, for the treatment of dogs with recurrent (either local or distant) mast cell tumor following surgical excision," Clinical Cancer Research, vol. 15, no. 11, pp. 3856-3865, 2009.

[5] M. Kiupel, J. D. Webster, K. L. Bailey et al., "Proposal of a 2-tier histologic grading system for canine cutaneous mast cell tumors to more accurately predict biological behavior," Veterinary Pathology, vol. 48, no. 1, pp. 147-155, 2011.

[6] C. L. R. Jones, R. A. Grahn, M. B. Chien, L. A. Lyons, and C. A. London, "Detection of $c$-kit mutations in canine mast cell tumors using fluorescent polyacrylamide gel electrophoresis," Journal of Veterinary Diagnostic Investigation, vol. 16, no. 2, pp. 95-100, 2004.

[7] R. Zavodovskaya, M. B. Chien, and C. A. London, "Use of kit internal tandem duplications to establish mast cell tumor clonality in 2 dogs," Journal of Veterinary Internal Medicine, vol. 6, pp. 915-917, 2004.

[8] J. D. Webster, V. Yuzbasiyan-Gurkan, J. B. Kaneene, R. Miller, J. H. Resau, and M. Kiupel, "The role of $c$-kit in tumorigenesis: evaluation in canine cutaneous mast cell tumors," Neoplasia, vol. 8, no. 2, pp. 104-111, 2006. 

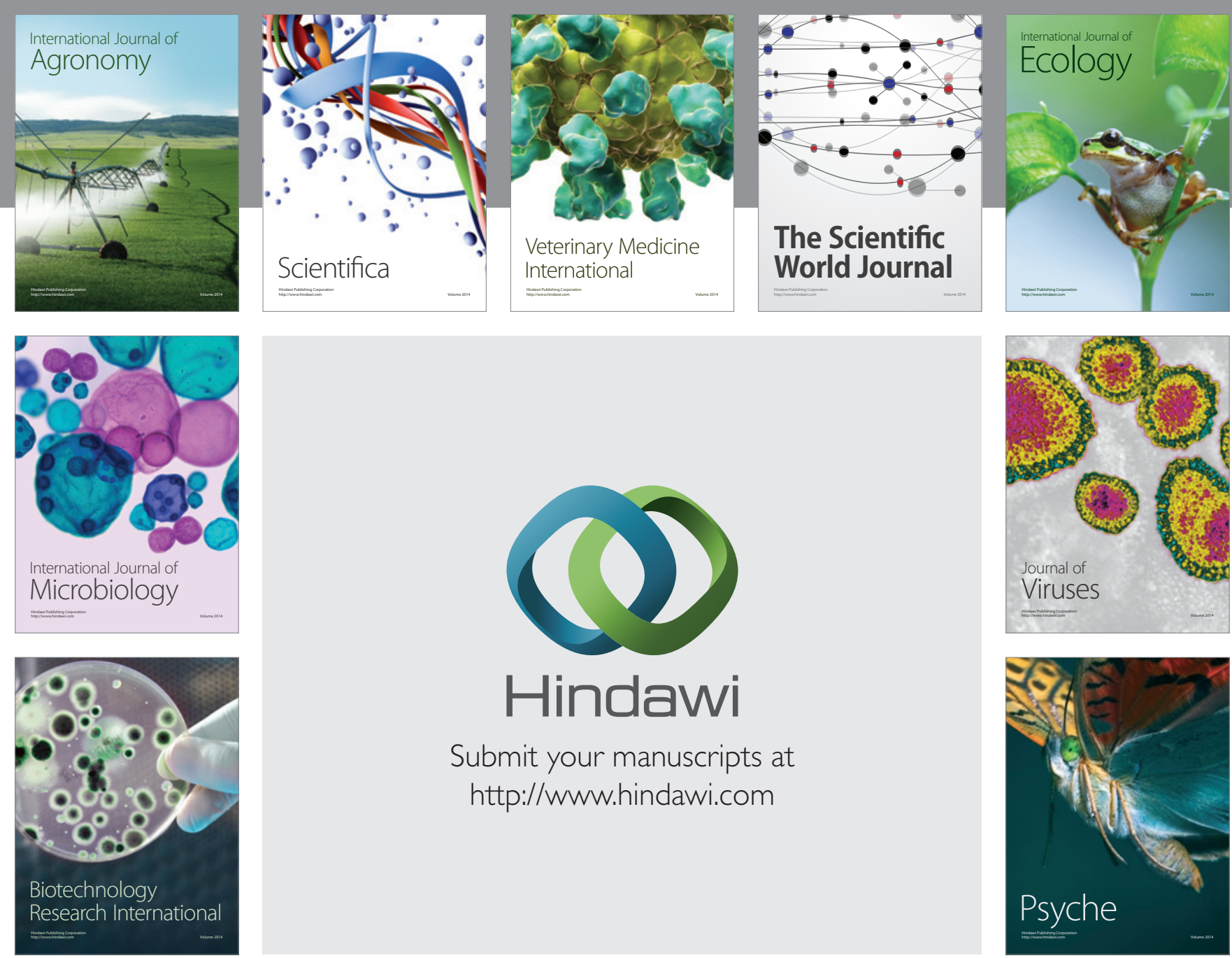

Submit your manuscripts at http://www.hindawi.com
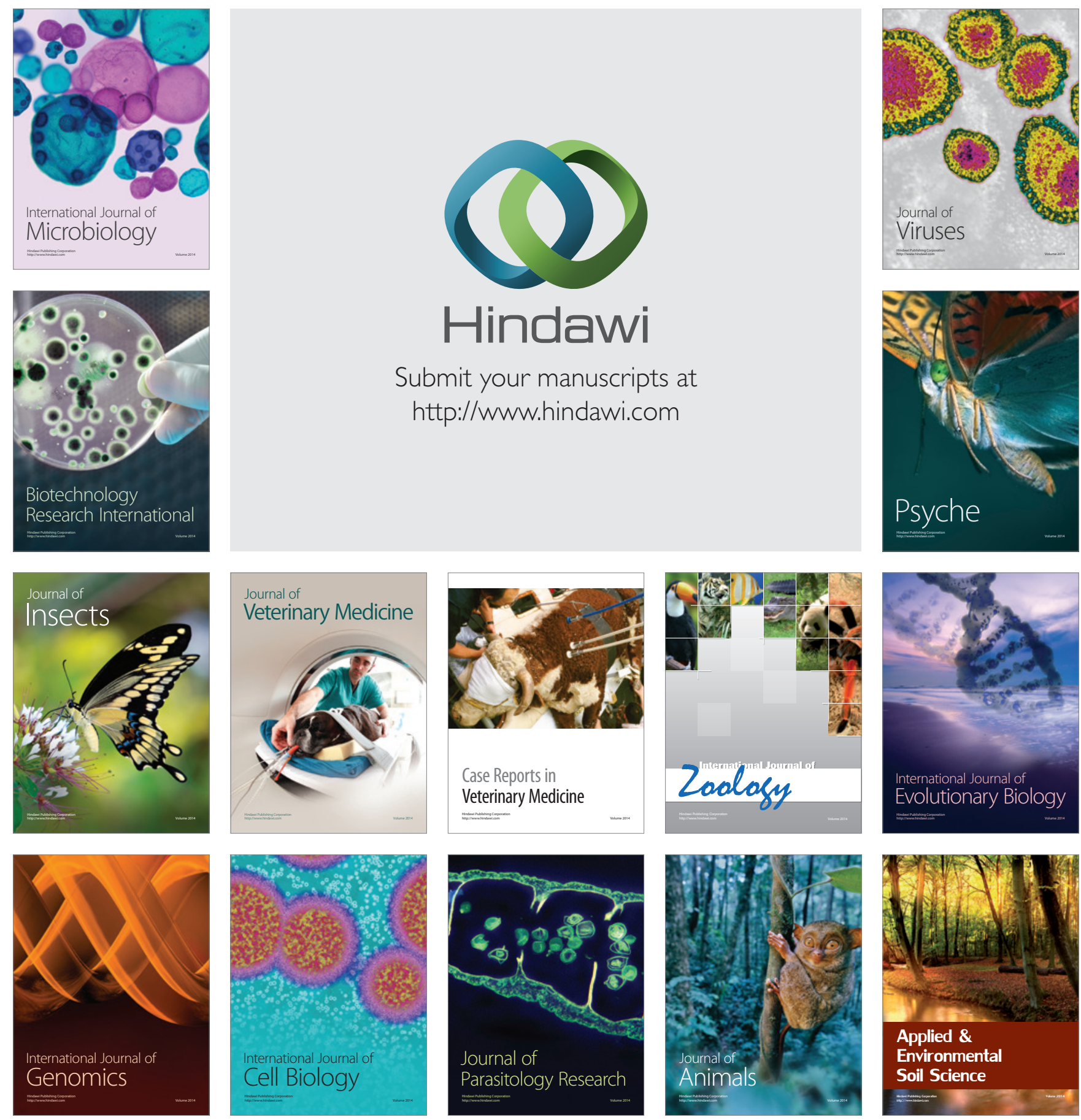